\title{
Loss and Temperature Computation of Damper Bars at Asymmetric Load Condtions of a Large Tubular Hydro-Generator
}

\author{
Lan Xiao ${ }^{1, a}$, Zhen-nan Fan ${ }^{2, b_{*}}$ \\ ${ }^{1}$ Technology \& Skill Training Center of Sichuan Electric Power Corporation, Chengdu China,610072 \\ ${ }^{2}$ Electrical Engineering College of Chongqing University, Chongqing, China, 400044 \\ axiaolan_sc@126.com, ${ }^{\mathrm{b}}$ mememf@126.com
}

Keywords: tubular hydro-generator, damper bar, loss and temperature, asymmetric load

\begin{abstract}
In order to research the loss and heat of damper bars on the asymmetric load condtions, a multi-slice moving electromagnetic field-circuit coupling FE model of tubular hydro-generator and a 3D temperature field FE model of the rotor are built respectively. And the loss and heat of damper bars at the asymmetric load condtions are computed and analyzed by these modles. The research is helpful for improving design standard of the large hydro-generator.
\end{abstract}

\section{Introduction}

Compared with the axial flowing hydro-generator with the same capability,the tubular hydro-generator can economize the project investment 10-25\% and increase 3-5\% of the power every year,so it has then been applied widely at the hydropower stations whose water head is lower than 20m [1]. However, the electromagnetic and cooling designs of the tubular hydro-generators are more difficult because of its limited inner space and then the more possibility of damper bars over heat[2]. In order to improve the generator design and avoid this failures, it's necessary to do the in-depth researches of the losses and heat of damper bars at the asymmetric load condtions.

The nonlinear electromagnetic field and eddy current are calculated to get the loss and heat in reference [3] [4] [5]. The temperature field of rotor are calculated in the 3D FE model in reference [6][7][8]. And the rotor temperature distribution of hydro-generator is calculated in fluid and temperature field method in reference [9] [10] [11].

But in general, for the tubular hydro-generator which is low number of slot per pole per phase (the number is always fractional slot, and the denominator is 2), and air gap is small, so far, there are few literature mentioned the in-depth study of the losses and heat of its damper bars at the asymmetric load condtions.

In this paper, for a 32MW tubular hydro-generator, a multi-slice moving electromagnetic field-circuit coupling FE model of the tubular hydro-generator and a 3D temperature field FE model of the rotor are built respectively. Then the the damper bar loss and heat at the asymmetric load condtions are computed and analyzed by these models.

\section{Calculation models}

\section{The basic data of the tubular hydro-generator}

The basic data and operate condtions of the generator are showed in Table 1 and Table 2 respectively .

\begin{tabular}{ll}
\multicolumn{2}{c}{ Table 1 The basic data of the generator } \\
\hline Parameter & Value \\
\hline Rated power $(\mathrm{MW})$ & 32 \\
Rated voltage $(\mathrm{kV})$ & 10.5 \\
Rated current $(\mathrm{A})$ & 1955 \\
Power factor & 0.9 \\
Number of magnetic poles & 76 \\
Stator slots skewed & 0.5 slot \\
\hline
\end{tabular}




\section{Boundary value problem of moving electromagnetic field}

\begin{tabular}{ll}
\hline Number of slots per pole per phase & $1 \frac{1}{2}$ \\
\hline \multicolumn{2}{c}{ Table 2 The operate condtions of the generator } \\
\hline Operate condtions \\
\hline 1 & Rated load \\
2 & Rated load with 6\% negative current \\
3 & Rated load with 12\% negative current \\
\hline
\end{tabular}

According to the periodicity of magnetic field, the area of a pair of poles is chosen as the electromagnetic field calculation region. And along the axial z, the generator is divided into 12 slices, as shown in Fig. 1.

Considering the saturation of iron core, the governing equation of nonlinear time-varying moving electromagnetic field is :

$$
\nabla \times(\nu \nabla \times \mathbf{A})+\sigma\left[\frac{\partial \mathbf{A}}{\partial t}-\mathbf{V} \times(\nabla \times \mathbf{A})\right]=\mathbf{J}_{\mathrm{s}}
$$

where $\mathbf{A}$ is vector magnetic potential, $\mathbf{J}_{\mathbf{s}}$ is source current density, $v$ is reluctivity, $\mathbf{V}$ is velocity and $\sigma$ is conductivity.

In the multi-slice moving electromagnetic field model, for each slice, the current density and vector magnetic potential have only the axial $\mathrm{z}$ components, and the speed has only the axial $\mathrm{x}$ component. By coulomb norm $\nabla \cdot \mathbf{A}=0$ and the boundary condition of the problem region, the 2D boundary value problem of nonlinear time-varying moving electromagnetic field for the generator is then obtained:

$$
\left\{\begin{array}{l}
\frac{\partial}{\partial x}\left(v \frac{\partial A_{s l z}}{\partial x}\right)+\frac{\partial}{\partial y}\left(v \frac{\partial A_{s l z}}{\partial y}\right)=-J_{s l z}+\sigma \frac{\partial A_{s l z}}{\partial t}+V_{x} \sigma \frac{\partial A_{s l z}}{\partial x} \\
\left.A_{s l z}\right|_{a r c_{-} i n}=\left.A_{s l z}\right|_{a r c_{0} \text { out }}=0 \\
A_{s l z} z_{\text {cyclic_boundary_start }}=\left.A_{s l z}\right|_{\text {cyclic_boundary_end }} .
\end{array}\right.
$$

Where $V_{x}$ is the axial $x$ component of velocity, $J_{s l z}$ is the axial $z$ component of source current density; $A_{s l z}$ is the axial $z$ component of vector magnetic potential.
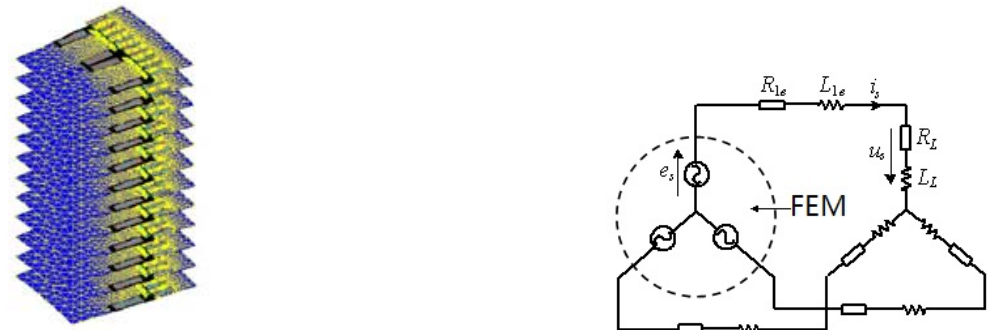

Fig. 1 The problem region and meshes of electromagnetic field

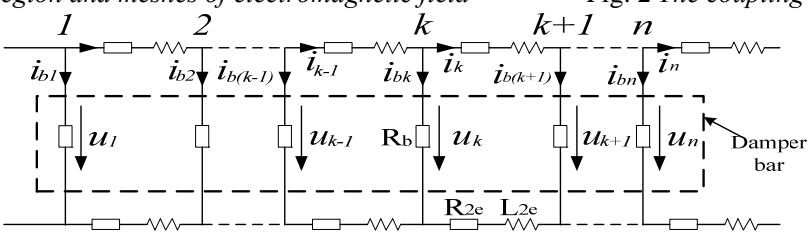

Fig. 3 The coupling circuit of the damper winding

\section{Coupling circuits}

To consider the influence of the end winding of the stator and damper end rings of rotor, the coupling circuit models are established.

The coupling circuit of stator and damper winding are shown in Fig. 2 and Fig.3.And the external circuit equation and electromagnetic equation should be combined in the calculation, then the flux density, current and loss can be got.

\section{Boundary value problem of rotor 3D temperature field}

Considered of the anisotropic heat conduction condition of the rotor core, the boundary value problem of 3D steady temperature field can be expressed as follows: 


$$
\left\{\begin{array}{l}
\frac{\partial}{\partial x}\left(\lambda_{x} \frac{\partial T}{\partial x}\right)+\frac{\partial}{\partial y}\left(\lambda_{y} \frac{\partial T}{\partial y}\right)+\frac{\partial}{\partial z}\left(\lambda_{z} \frac{\partial T}{\partial z}\right)=-q_{V} \\
\left.\lambda \frac{\partial T}{\partial n}\right|_{S_{2}}=0 \\
\left.\lambda \frac{\partial T}{\partial n}\right|_{S_{3}}=-\alpha\left(T-T_{f}\right)
\end{array}\right.
$$

where $T$ is temperature, $\lambda_{x}, \lambda_{y}$ and $\lambda_{z}$ are heat conductivity on each direction, $q_{v}$ is the heat source density which is obtained by losses calculation mentioned above, $S_{2}$ are the rotor middle profile and the interface between rotor core and rim related with the thermal insulation boundary condition, $S_{3}$ are the outside surfaces of the rotor related with the heat dissipation boundary condition, $\alpha$ is the heat dissipation coefficient of $S_{3}$ and $T_{f}$ is the environmental air temperature. The problem regions are illustrated in Fig. 4.

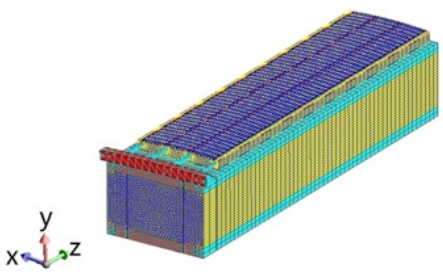

Fig. 4 The problem region and meshes of $3 D$ temperature field

\section{Computation results and discussions}

\section{The analyze for the heat of damper bars}

When the generator operates at the different conditions, the results of losses and heat of damper bars are listed in Table 3 . and some results are present in Fig.6. There are 4 damper bars on each pole shoe. For easy discussion of the computing results, the damper bar on the lee side is numbered $1^{\text {th }}$ and the damper bar on the windward is numbered $4^{\text {th }} . \mathrm{P}_{1}-\mathrm{P}_{4}$ and $\sum \mathrm{P}$ are the loss of the $1^{\text {st }}-4^{\text {th }}$ damper bar and the total losses of the damper bars respectively, $T_{\max }$ and $T_{\min }$ are the maximum and minimal temperature of damper bars.

Table 4 and Fig. 6show that the losses and heat of the $1^{\text {st }}$ and $2^{\text {nd }}$ damper bar which are on the lee side are significantly larger than those of $3^{\text {rd }}$ and $4^{\text {th }}$ damper bar which are on the windward. Because of the armature reaction, the distribution of the airgap magnetic field is distorted when the generator operates with rated load and asymmetric load. The magnetic field on the windward is weakened while it is strengthened on the lee side. Then the eddy current and loss on the lee side are significantly larger than those on the windward side. Besides, the dissipation condition on the lee side is weak than that on the windward, and the dissipation condition on the middle profile is weak than that on the end profile. Then the maximum temperature is at the middle of damper bar which near the lee side of the rotor pole.

And these results show that the loss and the temperature of the damper bars increase obviously when the operating conditions of the generator change from rated load to asymmetric rated load with $6 \%$ negative sequence current and then to asymmetric rated load with $12 \%$ negative sequence current. The loss and temperature of the damper bars for $12 \%$ negative sequence current are maximal respectively. The maximal temperature with this condition is 1.14 times higher than that with rated load, that is to say the temperature increases $23{ }^{\circ} \mathrm{C}$. The results show that the asymmetric load operating conditions increase the loss and heat of the damper bars obviously.

\begin{tabular}{llllllll}
\multicolumn{2}{l}{ Table 3 Losses and Temperatures of damper bars for different operate condtions } \\
\hline Operate & \multicolumn{1}{l}{ Loss $(\mathrm{W})$} & & & \multicolumn{3}{c}{ Temperature $\left({ }^{\circ} \mathrm{C}\right)$} \\
\cline { 2 - 8 } condtion & $P_{1}$ & $P_{2}$ & $P_{3}$ & $P_{4}$ & $\sum P$ & $T_{\max }$ & $T_{\min }$ \\
\hline 1 & 302 & 268 & 80 & 66 & 716 & 168 & 68 \\
2 & 340 & 290 & 93 & 79 & 802 & 177 & 69 \\
3 & 396 & 338 & 112 & 90 & 936 & 191 & 71 \\
\hline
\end{tabular}




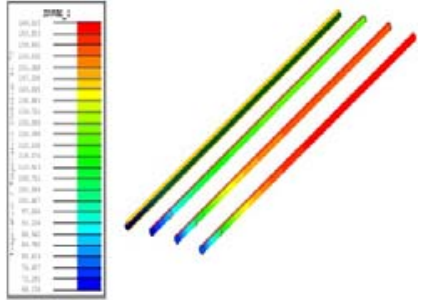

(a) Rated load

Fig. 6 Temperature distributions of damper bars with different operate condtions

\section{Verification of computation results}

In order to verify the correctness of the loss and heat calculation, the temperature test of the field winding is carried out to rated load condtion. The environmental air temperature is $45.6^{\circ} \mathrm{C}$ and the average temperature of field winding tested is $103^{\circ} \mathrm{C}$.The calculated average temperature of field winding is $97^{\circ} \mathrm{C}$ and is well agreed with the test dada.

\section{Conclusion}

In this paper,the multi-slice moving electromagnetic field-circuit coupling model of the hydro-generator and 3D temperature field FE model of rotor are implemented, the factors such as time-varying, moving, nonlinear, slot skew, eddy current of conduct are considered. It can forecast the influences of different operate condtions on the loss and heat of damper bars.

\section{References}

[1] Chao-Yang Li, "The Application of Bulb-type Hydro-Generator Set at Low Head Hydropower Station,” Developing, no. 9, pp. 145-146, 2006.

[2] Jing-Bin Guo, "Analysis of Damaged Damping Winding and Magnetic Pole in Bulb Type Generator,” Chinese Power, vol. 34, no. 7, pp. 63-67, May 2001.

[3] Du Yong, Cheng Zhi-guang, Yan Wei-li, "Electromagnetic Properties of Solid and Laminated Steel Using the Benchmark Models, " High Volt age Engineering, vol. 36, no. 5, pp. 1199-1204, May 2010.

[4] ZHAO Zhi-gang, LIU Fu-gui, CHENG Zhi-guang, "Loss and Flux Distribution of Power Transformer Laminated Core Under DC-biased Magnetization Condition in HVDC, " High Volt age Engineering, vol. 36, no. 9, pp. 2346-2351, September 2010.

[5] LI Hong-zhi, CUI Xiang1, LIU Dong-sheng, "Magnetic Circuit Modelling of the DC Biased Large Power Transformer and the Application, " High Volt age Engineering, vol. 36, no. 4, pp. 1068-1076, April 2010.

[6] Wei-Li Li, Feng Zhou, Yun-Peng Hou, and Shu-Kang Cheng, "Calculation of Rotor Temperature Field for Hydro-generator as well as the Analysis on Relevant Factors," Proceedings of the Chinese Society for Electrical Engineering, vol. 22, no. 10, pp. 85-90, October 2002.

[7] Jia-Bin Wen , Da-Wei Meng, and Chang-Bin Lu, "Synthetic Calculation for the Ventilation and Heating of Large Water Wheel Generator," Proceedings of the Chinese Society for Electrical Engineering, vol. 20, no. 11, pp. 6-9, November 2000.

[8] Jia-Bin Wen, Da-Wei Meng, Mei-Lan Zhou, and Chang-Bin Lu, "Field Model Research of Ventilation and Heat and Optimal Calculation of Ventilation Structure for Large Water Wheel Generator," Transactions of China Electrotechnical Society, vol. 15, no. 6, pp. 1-4+64, December 2000.

[9] Xia Hai-xia, Yao Ying-ying, Ni Guang-zheng, “Analysis of ventilation fluid field and rotor temperature field of a generator," Electric Machines and Control, vol. 11, no. 5, pp. 472-476, October 2007.

[10] Xu Xu, Xu Hong, Li tao, "Ventilation and stator temperature field in a vertical hydro power generator,” Journal of Engineering Thermophysics, vol. 30, no. 10, pp. 1717-1719, October 2009. 